\title{
PSEUDO-IDEMPOTENTS IN SEMIGROUPS OF FUNCTIONS
}

FRANK A. CEZUS

(Received 10 April 1972)

Communicated by G. B. Preston

The aim of this paper is to generalize Theorem 2.10 (i) of [2]. As stated in [2] this theorem deals with the semigroup of all selfmaps on a discrete space and provides a characterization of $\mathscr{H}$-classes which contain an idempotent. We will generalize this theorem to the case of other semigroups of functions on a discrete space, some semigroups of continuous functions on non-discrete topological spaces, and one semigroup of binary relations. The results in this paper form the main part of chapter 3 of [1]. Some results will be quoted from [1] without proof; the required proofs can easily be supplied by the reader.

Notation for composition of functions will be written in topologicalanalytic order: $(f g)(x)=f(g(x))$. Thus the concepts of left and right in this paper will be the mirror images of left and right in [2]. Juxtaposition will always denote ordinary composition. Definition 4 will be concerned with a semigroup multiplication which is not ordinary composition.

We will let $\operatorname{Dom}(f)$ denote the domain of a function $f$, and $\operatorname{Im}(f)$ will denote the image. The equivalence class of $f$ under a Green's relation, say $\mathscr{L}$, will be called an $\mathscr{L}$-class and will be written $L_{f}$.

Definition 1. a. $\pi_{f}=\pi_{g}$ means that $\operatorname{Dom}(f)=\operatorname{Dom}(g)$ and that for arbitrary $x$ and $y$ in $\operatorname{Dom}(f)=\operatorname{Dom}(g)$ we have $f(x)=f(y)$ if and only if $g(x)=g(y)$.

b. A semigroup $T$ is said to be $L_{\pi}$ if for arbitrary $f$ and $g$ in $T$ we have $L_{f}=L_{g}$ if and only if $\pi_{f}=\pi_{g}$.

c. A semigroup $T$ is said to be $R_{i m}$ if for arbitrary $f$ and $g$ in $T$ we have $R_{f}=R_{g}$ if and only if $\operatorname{Im}(f)=\operatorname{Im}(g)$.

Definition 2. Let $X$ be a topological space.

a. $S(X)$ is the semigroup of all continuous functions from all of $X$ into $X$ under ordinary composition.

b. $S_{1}(X)$ is the subsemigroup of one-to-one functions in $S(X)$.

c. $Q(X)$ is the semigroup of all continuous functions from $X$ into $X$ whose domains are open subsets of $X$. Multiplication is ordinary composition. 
d. $Q_{1}(X)$ is the subsemigroup of one-to-one functions in $Q(X)$.

From the above definition we see that $S_{1}(X)=S(X) \cap Q_{1}(X)$, while $Q(X)$ can be thought of as the smallest semigroup containing $S(X)$ and $Q_{1}(X)$. We note that $S(X)$ with $X$ discrete is an $L_{\pi}$ and $R_{i m}$ semigroup according to Lemmas 2.5 and 2.6 of [2]. It is easy to see that for $X$ discrete the semigroups $Q(X)$ and $Q_{1}(X)$ are also $L_{\pi}$ and $R_{i m}$; however, $S_{1}(X)$ is $R_{i m}$ but not $L_{\pi}$ for infinite discrete $X$.

In the following three lemmas we assume that $\operatorname{Dom}(f)$ and $\operatorname{Im}(f)$ are subsets of a topological space $X$.

LEMMA 1. The following conditions are equivalent:

i) $\operatorname{Im}(f f)=\operatorname{Im}(f)$;

ii) $f^{-1}(f(x)) \cap \operatorname{Im}(f) \neq \varnothing$ for each $x \in \operatorname{Dom}(f)$.

Proof. $\mathrm{i} \rightarrow$ ii. For each $x \in \operatorname{Dom}(f)$ we have $f(x)=f f(z)$ for some $z$. Since

$$
f(z) \in f^{-1}(f f(z))=f^{-1}(f(x))
$$

we see that $f(z) \in f^{-1}(f(x)) \cap \operatorname{Im}(f) \neq \varnothing$.

ii $\rightarrow$ i. Clearly $\operatorname{Im}(f f) \subset \operatorname{Im}(f)$. Let $y \in \operatorname{Im}(f)$ be arbitrary; $y=f(x)$. Let

$$
z \in f^{-1}(f(x)) \cap \operatorname{Im}(f) .
$$

Then $z=f(t)$ for some $t$, and hence $y=f(x)=f(z)=f f(t) \in \operatorname{Im}(f f)$.

LEMMA 2. The following conditions are equivalent:

i) $\operatorname{Im}(f f)=\operatorname{Im}(f)$ and $\left.f\right|_{\operatorname{Im}(f)}$ is one-to-one;

ii) $f^{-1}(f(x)) \cap \operatorname{Im}(f)$ is a single point for each $x \in \operatorname{Dom}(f)$.

Proof. i) $\rightarrow$ ii). By the previous lemma we know that $f^{-1}(f(x)) \cap \operatorname{Im}(f) \neq \varnothing$. Let $y$ and $z$ be in $f^{-1}(f(x)) \cap \operatorname{Im}(f)$. Then $f(y)=f(x)$ and $y=f(s)$ for some $s$; also $f(z)=f(x)$ and $z=f(t)$ for some $t$. Therefore $f(y)=f(z)$, and thus $f f(s)=f f(t)$. Since $f$ is one-to-one on $\operatorname{Im}(f)$ we have $f(s)=f(t)$, that is, $y=z$.

ii) $\rightarrow$ i). By the previous lemma we know that $\operatorname{Im}(f f)=\operatorname{Im}(f)$. Suppose that $y$ and $z$ are in $\operatorname{Im}(f)$ with $f(y)=f(z)$. Then $y$ and $z$ are in $f^{-1}(f(y)) \cap \operatorname{Im}(f)$, and thus $y=z$.

We note that in condition i) of the preceding lemma we do not assume that $\operatorname{Im}(f) \subset \operatorname{Dom}(f)$. If in fact we have $\operatorname{Im}(f) \subset \operatorname{Dom}(f)$, then condition i) says precisely that $f$ is a permutation on $\operatorname{Im}(f)$. Condition ii) of the lemma enables us to define a function

$$
g(x) \equiv f^{-1}(f(x) \cap \operatorname{Im}(f) \text { on } \operatorname{Dom}(f) .
$$

This function is examined in the following lemma.

Lemma 3. Suppose $\operatorname{Im}(f) \subset \operatorname{Dom}(f)$ where $f$ is a function which satisfies 
the equivalent conditions in the preceding lemma. Define $g(x) \equiv f^{-1}(f(x)) \cap \operatorname{Im}(f)$ for each $x \in \operatorname{Dom}(f)$. Then $\pi_{f}=\pi_{g} ; \operatorname{Im}(f)=\operatorname{Im}(g)$; and $g g=g$.

Proof. It is clear that $\operatorname{Dom}(f)=\operatorname{Dom}(g)$. Suppose $f(x)=f(y)$. Then $g(x)=g(y)$ by inspection. On the other hand, suppose $f(x) \neq f(y)$. Then

$$
f^{-1}(f(x)) \cap f^{-1}(f(y))=\varnothing
$$

and therefore $g(x) \neq g(y)$. Hence $\pi_{f}=\pi_{g}$. Now let $y \in \operatorname{Im}(f)$. Then

$$
g(y)=f^{-1}(f(y)) \cap \operatorname{Im}(f)=y .
$$

Therefore $\left.g\right|_{\operatorname{Im}(f)}=\left.i\right|_{\operatorname{Im}(f)}$. Thus $\operatorname{Im}(f) \subset \operatorname{Im}(g)$, but by definition we have $\operatorname{Im}(g) \subset \operatorname{Im}(f)$. Consequently $\operatorname{Im}(f)=\operatorname{Im}(g)$ and $\left.g\right|_{\operatorname{Im}(g)}=\left.i\right|_{\operatorname{Im}(g)}$, that is, $g g=g$.

In view of Lemmas 2 and 3 we now define pseudo-idempotency in $S(X)$, $S_{1}(X), Q(X)$, and $Q_{1}(X)$.

Definition 3. Let $T$ be a semigroup of the form $S(X), S_{1}(X), Q(X)$, or $Q_{1}(X)$ for some topological space $X$. We say that a function $f \in T$ is pseudoidempotent if $\operatorname{Im}(f f)=\operatorname{Im}(f) \subset \operatorname{Dom}(f)$ and $\left.f\right|_{I \mathrm{~m}(f)}$ is one-to-one, that is, $f$ is a permutation on $\operatorname{Im}(f)$.

We remark that in $S_{1}(X)$ the only idempotent is the identity function, and the pseudo-idempotents are precisely the onto functions. In $S(X)$ and $S_{1}(X)$ the condition $\operatorname{Im}(f) \subset \operatorname{Dom}(f)$ is superfluous. In $Q_{1}(X)$ it is easy to see that $\operatorname{Im}(f)=\operatorname{Dom}(f)$ for a pseudo-idempotent $f$. On the the basis of Definition 3 we can state the central theorem of this paper.

THEOREM 1. Let $H$ be an $\mathscr{H}$-class in $S(X), S_{1}(X), Q(X)$, or $Q_{1}(X)$ with $X$ discrete. The following conditions are equivalent:

1) $H$ contains a pseudo-idempotent;

2) $H$ contains an idempotent (unique);

3) $H$ consists of pseudo-idempotents.

Proof. 1) $\rightarrow 2$ ). This follows from Lemma 3 together with the remarks preceding Lemma 1 and following Definition 3. Uniqueness of the idempotent follows from Lemma 2.15 of [2].

$2) \rightarrow 3$ ). Let $f$ be the idempotent in $H$, and let $g \in H$ be arbitrary. Then $\pi_{f}=\pi_{g}$ and $\operatorname{Im}(f)=\operatorname{Im}(g)$, and it is easy to check therefore that the conditions in Lemma 2 hold for $g$ and that $\operatorname{Im}(g) \subset \operatorname{Dom}(g)$.

3) $\rightarrow$ 1). Trivial.

We will now establish the results of Theorem 1 for some semigroups of the form $S(X)$ and $S_{1}(X)$ where $X$ is a non-discrete topological space. Similar results 
can be obtained for $Q(X)$ and $Q_{1}(X)$. In order to establish Theorem 1 with $X$ non-discrete we only have to verify the implication $1 \rightarrow 2$. To do this we must show that the idempotent

$$
g(x) \equiv f^{-1}(f(x)) \cap \operatorname{Im}(f)
$$

is continuous and belongs to the $\mathscr{H}$-class of $f$.

Suppose, for instance, that $X$ is a compact Hausdorff space for which $S(X)$ is an $L_{\pi}$ and $R_{i m}$ semigroup. These assumptions hold, for example, for $X$ finite discrete or $X$ equal to $\{0\} \cup\{1 / n\}_{n=1,2, \ldots}$ with the usual metric topology (see [1], Propositions 2.16 and 2.17). Then for each pseudo-idempotent $f \in S(X)$ the corresponding idempotent $g$ is continuous because $X$ is compact Hausdorff (see [1], Lemma 3.8), and $g \in H_{f}$ by Lemma 3 above. Therefore Theorem 1 holds for $S(X)$ in this case.

Let $I$ be the closed unit interval, and consider $S(I)$. By Lemma 3.8 of [1] we know that the idempotent $g$ is continuous. Since $S(I)$ is an $L_{\pi}$ semigroup (see [1], Proposition 2.23) we know that $g \in L_{f}$ by Lemma 3 above. $S(I)$ is not an $R_{i m}$ semigroup, but it is shown in Corollary 3 of Theorem 3.2 of [1] that $g \in R_{f}$. Hence Theorem 1 holds for $S(I)$. We can also show that Theorem 1 holds for $S(R)$ where $R$ is the real line (see [1], Corollary 4 of Theorem 3.2).

For semigroups of the form $S_{1}(X)$ the situation depends on whether any onto functions $f \in S_{1}(X)$ fail to be invertible in $S_{1}(X)$. As we remarked after Definition 3, the semigroup $S_{1}(X)$ contains only one idempotent, the identity function $i$; and therefore Theorem 1 is concerned with $H_{i}$. Clearly $H_{i}$ in $S_{1}(X)$ for any $X$ consists of the continuously invertible onto functions. The pseudoidempotents are the onto functions. We conclude that Theorem 1 holds for $S_{1}(X)$ if and only if each onto function in $S_{1}(X)$ is continuously invertible. It is then clear that Theorem 1 holds, for instance, for $S_{1}(X)$ with $X$ equal to an interval of the real line or equal to the space $p=\{0\} \cup\{1 / n\}$ with the metric topology. For $P N=\{0\} \cup\{1 / n\} \cup\{n\}$ we can see that Theorem 1 is false for both $S_{1}(P N)$ and $S(P N)$.

Finally we will establish the results of Theorem 1 for some semigroups which are not included in Definition 2.

Definition 4. a. Let $Y$ be a subspace of $X$. Then $S(X, Y)$ is the subsemigroup of functions $f \in S(X)$ such that $f(Y) \subset Y$.

b. Let $X$ and $Y$ be arbitrary topological spaces. Let $p$ te a contincous function which maps all of $Y$ into $X$. Then $S(X, p, Y)$ is the semigroup of all continuous functions which map all of $X$ into $Y$ under the multiplication $f \circ g \equiv f p g$.

c. Let $X$ be an arbitrary set. For a binary relation $T$ on $X$ we let $T(x)$ $=\{y \mid x T y\}$. Then $B_{1}(X)$ denotes the semigroup of all binary relations $T$ on $X$ such that $x \neq y$ implies $T(x) \cap T(y)=\varnothing$. 
The semigroups $S(X, Y)$ were called restrictive semigroups by Magill in [3], and the semigroups $S(X, p, Y)$ were discussed by Magill in [4].

First we will establish Theorem 1 for $S(X, Y)$ with $X$ discrete. The main task is to relate the definition of pseudo-idempotency in $S(X, Y)$ to the subspace $Y$. To this end we observe that if $f \in S(X, Y)$ is idempotent, then each of the functions $\left.f\right|_{\operatorname{Im}(f)},\left.f\right|_{Y_{\cap \operatorname{Im}(f)}}$, and $\left.f\right|_{f(Y)}$ is the identity on its domain. We define a function $f \in S(X, Y)$ to be pseudo-idempotent if each of the functions $\left.f\right|_{\operatorname{Im}(f)}$, $\left.f\right|_{Y \cap \operatorname{Im}(f)}$, and $\left.f\right|_{f(Y)}$ is a permutation. Given a pseudo-idempotent, we then define the corresponding idempotent exactly as we did in Lemma 3. In order to show that the idempotent $g$ is in the $\mathscr{H}$-class of the pseudo-idempotent $f$ we first have to characterize $\mathscr{H}$-classes in $S(X, Y)$ with $X$ discrete. We define the symbol $f Y g$ to mean that for each $x \in X$ we have $f(x) \in Y$ if and only if $g(x) \in Y$. Then we can show that $L_{f}=L_{g}$ if and only if $\pi_{f}=\pi_{g}$ and $f Y g$ (see [1], Proposition 2.40), and $R_{f}=R_{g}$ if and only if $\operatorname{Im}(f)=\operatorname{Im}(g)$ and $f(Y)=g(Y)$ (see [1], Proposition 2.41). With the resulting characterization of $\mathscr{H}$-classes it is easy to show that Theorem 1 is valid for $S(X, Y)$.

We will now consider $S(X, p, Y)$ for the case of $X$ and $Y$ discrete. Clearly a function $f \in S(X, p, Y)$ is idempotent if and only if $\left.f p\right|_{\operatorname{Im}(f)}=\left.i\right|_{\operatorname{Im}(f)}$. We then say that $f \in S(X, p, Y)$ is pseudo-idempotent if $\left.f p\right|_{\operatorname{Im}(f)}$ is a permutation. Given a pseudo-idempotent $f$ we construct the corresponding idempotent $g$ by the formula

$$
g(x) \equiv(f p)^{-1}(f(x)) \cap \operatorname{lm}(f) .
$$

With the help of the following lemma, which is Lemma 3.26 of [1], it can be shown that $g \in H_{f}$ and that Theorem 1 is valid for $S(X, p, Y)$.

Lemma 4. Let $X$ and $Y$ be discrete and let $f$ and $g$ be pseudo-idempotents in $S(X, p, Y)$. If $L_{p f}=L_{p g}$ in $S(X)$, then $L_{f}=L_{g}$ in $S(X, p, Y)$. If $R_{f p}=R_{f p}$ in $S(Y), R_{f}=R_{g}$ in $S(X, p, Y)$.

We will conclude by considering the semigroup of relations $B_{1}(X)$. For $T$ and $V$ in $B_{1}(X)$ we can show that $L_{T}=L_{V}$ if and only if $\operatorname{Dom}(T)=\operatorname{Dom}(V)$, and $R_{T}=R_{V}$ if and only if each $T(x)$ equals $V(y)$ for some $y$ and each $V(u)$ equals $T(w)$ for some $w$ (see [1], Propositions 5.1 and 5.2).

The definition of pseudo-idempotency in $B_{1}(X)$ will be based on the following lemma.

Lemma 5. A relation $T \in B_{1}(X)$ is idempotent if and only if $T(x) \cap \operatorname{Dom}(T)$ $=\{x\}$ for each $x \in \operatorname{Dom}(T)$.

By means of this lemma we have found an identity function, namely, $T(x) \cap \operatorname{Dom}(T)$ on $\operatorname{Dom}(T)$. We then define a relation $T \in B_{1}(X)$ to be pseudoidempotent if $T(x) \cap \operatorname{Dom}(T)$ is a singleton for each $x \in \operatorname{Dom}(T)$ and the resulting 
function $f(x) \equiv T(x) \cap \operatorname{Dom}(T)$ is a permutation on $\operatorname{Dom}(T)$. For $T \in B_{1}(X)$ let $T^{-1}(y)=\{x \mid x T y\}$, which is either a singleton or the empty set.

Lemma 6. Let $T \in B_{1}(X)$ be pseudo-idempotent. Define $V$ on $\operatorname{Dom}(T)$ by the formula $V(x) \equiv T\left(T^{-1}(x)\right)$. Then $V \in B_{1}(X) ; V$ is idempotent, and $H_{T}=H_{V}$.

Proof. To see that $V \in B_{1}(X)$ we suppose that $V(x) \cap V(y) \neq \varnothing$. Then

$$
T^{-1}(x) \cap T^{-1}(y) \neq \varnothing .
$$

Since $T^{-1}$ is a permutation on $\operatorname{Dom}(T)$, it follows that $x=y$, which completes the first part of the proof.

To see that $V$ is idempotent we consider the expression $T^{-1} T T^{-1}(x)$. Since $T$ is in $B_{1}(X)$ we have

$$
T^{-1} T T^{-1}(x)=T^{-1}(x) \text {. }
$$

Therefore $V V(x)=T\left(T^{-1} T T^{-1}(x)\right)=T T^{-1}(x)=V(x)$.

We will now show that $H_{T}=H_{V}$. By the definition of $V$ we have $\operatorname{Dom}(T)$ $=\operatorname{Dom}(V)$, and thus $L_{T}=L_{V}$. Since $T^{-1}$ is a permutation on $\operatorname{Dom}(T)$, we see from the definition of $V$ that each $V(x)$ equals $T(y)$ for some $y$, and each $T(z)$ equals $V(w)$ for some $w$. Therefore $R_{T}=R_{V}$, and the proof is done.

From this lemma it now follows easily that Theorem 1 is true for $B_{1}(X)$.

\section{References}

[1] F. Cezus, Green's Relations in Semigroups of Functions (Ph.D. thesis submitted May, 1972 at the Australian National University).

[2] A. H. Clifford and G. B. Preston, The Algebraic Theory of Semigroups (Math. Surveys, No. 7, Amer. Math. Soc., 1961 and 1967).

[3] K. D. Magill, Jr, 'Subsemigroups of $S(X)$ ', Math. Japonicae 11 (1966), 109-115.

[4] K. D. Magill, Jr, 'Semigroup structures for families of functions. I', J. Austral. Math. Soc. 7 (1967), 81-94.

The Australian National University

Canberra, A. C. T. 